\title{
Design and Analysis of an Optical Coupler for Concentrated Solar Light Using Optical Fibers in Residential Buildings
}

\author{
Afshin Aslian, ${ }^{1}$ Barmak Honarvar Shakibaei Asli, ${ }^{2,3}$ Chin Joo Tan, ${ }^{1}$ \\ Faisal Rafiq Mahamd Adikan, ${ }^{2}$ and Alireza Toloei ${ }^{4}$ \\ ${ }^{1}$ Department of Mechanical Engineering, Faculty of Engineering, University of Malaya, Lembah Pantai, \\ 50603 Kuala Lumpur, Malaysia \\ ${ }^{2}$ Integrated Lightwave Research Group, Department of Electrical Engineering, Faculty of Engineering, University of Malaya, \\ Lembah Pantai, 50603 Kuala Lumpur, Malaysia \\ ${ }^{3}$ Institute of Information Theory and Automation, Academy of Sciences of the Czech Republic, Pod Vodárenskou věži 4, \\ 18208 Praha, Czech Republic \\ ${ }^{4}$ Faculty of New Technologies Engineering, Shahid Beheshti University, District 1, Daneshjou Boulevard, Tehran 1983963113, Iran
}

Correspondence should be addressed to Chin Joo Tan; tancj@um.edu.my

Received 7 December 2015; Revised 7 April 2016; Accepted 21 April 2016

Academic Editor: Manuel Fuentes Conde

Copyright (C) 2016 Afshin Aslian et al. This is an open access article distributed under the Creative Commons Attribution License, which permits unrestricted use, distribution, and reproduction in any medium, provided the original work is properly cited.

\begin{abstract}
Concentrated sunlight that is transmitted by fiber optics has been used for generating electricity, heat, and daylight. On the other hand, multijunction photovoltaic cells provide high efficiency for generating electricity from highly concentrated sunlight. This study deals with designing and simulating a high-efficiency coupler, employing a mathematical model to connect sunlight with fiber optics for multiple applications. The coupler concentrates and distributes irradiated light from a primary concentrator. In this study, a parabolic dish was used as the primary concentrator, a coupler that contains nine components called a compound truncated pyramid and a cone (CTPC), all of which were mounted on a plate. The material of both the CTPC and the plate was BK7 optical glass. Fiber optics cables and multijunction photovoltaic cells were connected to the cylindrical part of the CTPC. The fibers would transmit the light to the building to provide heat and daylight, whereas multijunction photovoltaic cells generate electricity. Theoretical and simulation results showed high performance of the designed coupler. The efficiency of the coupler was as high as $92 \%$, whereas the rim angle of the dish increased to an optimum angle. Distributed sunlight in the coupler increased the flexibility and simplicity of the design, resulting in a system that provided concentrated electricity, heat, and lighting for residential buildings.
\end{abstract}

\section{Introduction}

Stand-alone or net-zero energy buildings are residential or commercial buildings with greatly reduced energy needs [1]. The consumed energy in a residential building can be in various forms, including light, heat, and electricity. Electric power generation from sunlight and its conversion to heat or light has a low efficiency. In concentrated photovoltaic systems, the temperature of the photovoltaic module and coupler increases; therefore, a cooling system is required. Moreover, concentrated photovoltaic and thermal (CPVT) systems demonstrate higher efficiency versus conventional thermal solar systems [2].
More than 30 years ago, a group of researchers studied the transport of solar energy via fiber optics [3]. This idea inspired other researchers because of many potential applications of this kind of solar system, including the production of passive daylight [4] and solar surgery [5] and using solar energy for heating or cooling [6, 7]. However, some disadvantages such as the cost of fiber optics cables [8], the effect of dust [9], and the high temperature of concentrated light on the tip of fibers [10-12] have prevented commercial use of the system. Such a system compromises a concentrator, a tracker for high concentration, a sunlight coupler, and fiber optics. Other devices similar to photovoltaic (PV) cells and heat exchangers may be added for use with different applications. Improvements 
in the components have encouraged researchers to attempt overcoming previous obstacles. Concentrators are generally grouped by optical characteristics, including low (less than 100x), medium (100x-300x), and high (more than 300x) concentrations. However, all proposed systems increase the intensity of light, and hence fewer PV cells or heating surfaces are required for generating electrical power or heat. This strategy will reduce the cost of the total system and land utilization [13]. High concentration is required for focusing sunlight into fiber optics. Parabolic mirror dishes are widely used in solar energy applications for highly concentrating the sunlight. The parabolic dish is an important subsystem that offers the highest thermal and optical efficiency among other options [14]. The cost of this component has a noticeable effect on the total cost of the system. In terms of scale, two approaches are proposed to gain the maximum efficiency of sunlight. Each approach alters the design of the coupler and other components differently. The first approach considers a solar dish with a diameter either equal to or larger than $1 \mathrm{~m}$ as a large dish, and it is far more expensive than a minidish (diameter less than $1 \mathrm{~m}$ ). In this case, the wind force significantly affects the design of the tracker and the structure of the system. Nevertheless, because it requires fewer components, the overall cost decreases in large-scale power plants using large dishes. Thus, novel designs have been introduced to reduce the cost of large-scale solar dishes in the last decade $[15,16]$.

Small dishes are proposed for high concentration and the generation of daylight as the second approach [17]. The spatial restrictions for installing large dishes on rooftops prompted designers to use minidishes or an array of minidishes for residential applications [18]. Coupling a premier concentrator with fiber optics and, simultaneously, increasing the concentricity of light with high efficiency have been a significant challenge. When a bundle of fiber optics is placed at the focal point of the dish, the tip of the fiber is exposed to intense heat. This is because the bundle has a total core area that is less than $50 \%$ of the cross-sectional area of the bundle. The concentrated sunlight, which propagates on the clad or gap of the bundle, will be converted to heat [10]. On the other hand, the acceptance angle of fibers is limited, and the concentrated sunlight has to be transmitted inside a small cone angle. Because of that, the portion of sunlight with an angle more than the critical angle is not transmitted by the fiber optic. Furthermore, concentrating high-intensity sunlight into a spot with tiny-diameter fiber optics is not practical; therefore, bundles of fibers have been used for transmitting sunlight [19]. However, using more fibers increased the cost. To tackle these problems, various systems and methods have been used to maximize the concentration and homogenization of the light. In 1996, Winston and Ning introduced a coupler, called a nonimaging radiant energy device, that included a convex lens at the inlet, a nonimaging hyperbolically shaped device, and a concave lens at the outlet [20].

In another study, a bundle of fibers transmitted sunlight while a parabolic dish was applied as the main concentrator. The coupler consisted of a secondary mirror and an aspheric lens [19]. Feuermann and Gordon worked on the usage of solar fiber optics in minidishes, and they presented a coupling with a single compound parabolic concentrator (CPC) that was placed in the dish and received the light reflected from a small flat mirror. The CPC collected sunlight and was coupled with the fiber optic cable [12]. They also used fiber as a direct receiver of light and compared it with the CPC coupler [21]. He et al. [22] designed and implemented a novel concentrator and coupler based on a solar fiber lamp. Their proposed design was composed of a concentrator with a compound curved surface and a coupler. The coupler was made of a cylindrical mirror surface, a deflector, a CPC, and fiber connected to the endpoint of the CPC.

Ullah and Shin studied highly concentrated optical fiberbased daylighting systems that used CPCs for coupling fibers with the generation of concentrated light by a primary concentrator [23]. Yu et al. [24] investigated the usage of miniature dielectric compound parabolic concentrators (DCPCs) for day lighting. Arnaoutakis et al. showed raytracing simulations of a novel two-stage coupler that had a lens and a dielectric taper of the circular cross section that was directly attached to the optical fiber [25].

This paper introduces a new coupler based on dividing sunlight using the proposed compound pyramid and cone shape implemented in a concentrated photovoltaic, thermal, and lighting system. Each CTPC can be connected to the core of one fiber. Therefore, the loss would be reduced, which leads to the reduction of temperature on the tips of the fiber optics. An analytical model was developed to design the components of this structure. The new design is capable of transmitting sunlight into fiber optics and working as a secondary concentrator. It also reduces the angle of incident light before entering the fiber optics while simultaneously increasing the coupling efficiency by reducing the number of optical components with different mediums. We divided the sunlight into nine beams, where each beam hits one CTPC with small angle of incidence. The designed CTPC facilitates the assembly process of fiber optics and coupler. The design of the coupler is simulated and the results are presented.

\section{Coupling Design, Theory, and Methodology}

Figure 1 shows a schematic of the proposed coupler, dish, and fiber optics. The coupler is attached to the dish and fiber optics. The dish is used as a primary concentrator, and the fiber optics transmit the sunlight.

The proposed coupler includes nine CTPCs mounted on a transparent plate. The coupler is placed a few millimeters from the focal point of the dish. Since the refractive index of the plate is higher than that of air, it reduces the refracted angle of light. Considering prototype methodology, two different concepts were developed. First, a prototyping process with a flat plate as the base of the coupler was assumed. In this case, the bottom of each CTPC was cut to a specific angle. In the second approach, one side of the plate was machined so that it could contain nine square surfaces with different angles. In this case, all the CTPCs were the same, which rendered them more suitable for mass production. As shown in Figure 2(a), according to the first concept, the surface of the bottom side of the CTPCs is rectangular in order to accommodate them on the flat plate with no empty space (see 


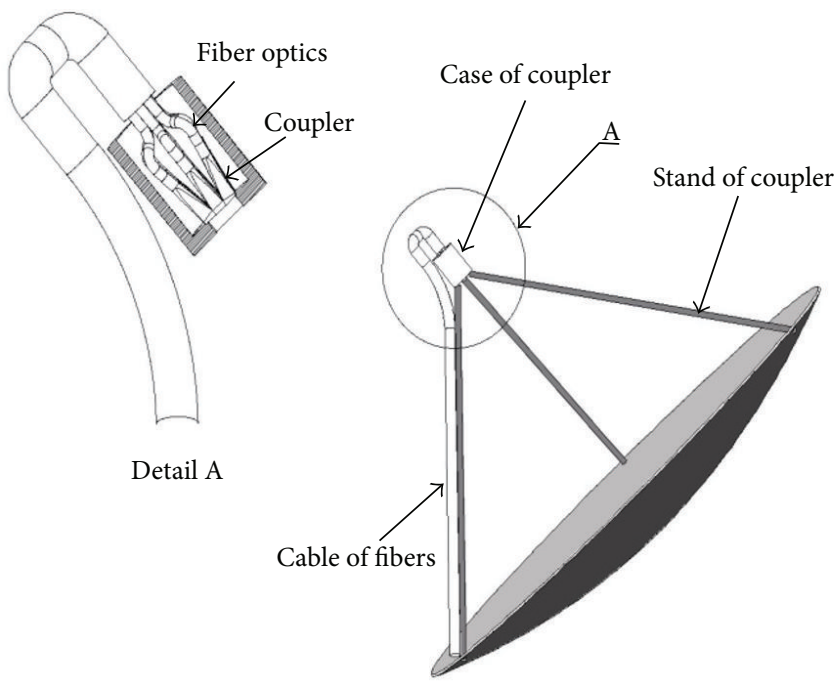

FIGURE 1: Schematic of a parabolic dish, coupler, and fiber optics.

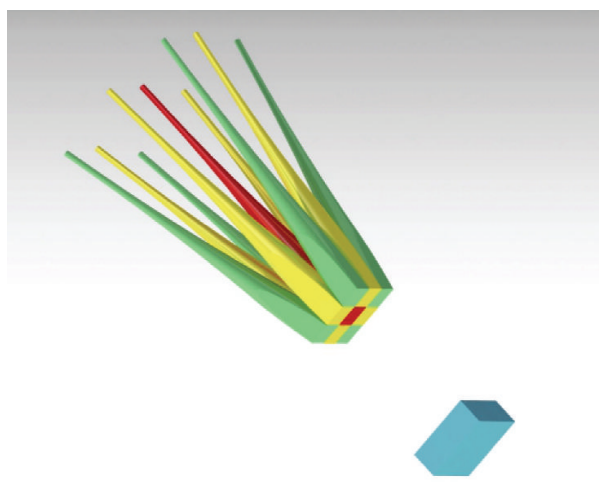

(a)

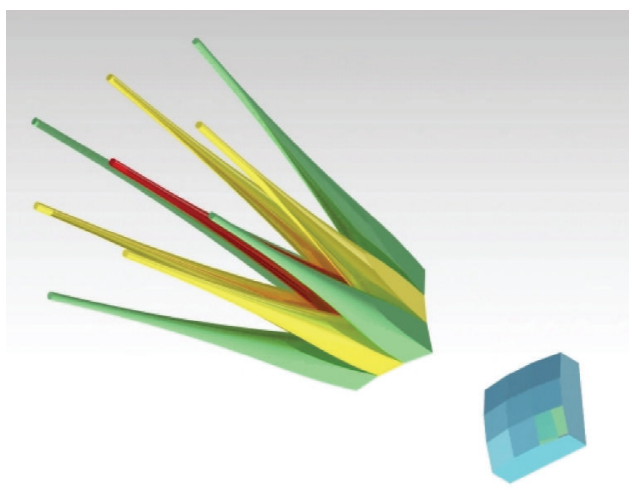

(b)

FIGURE 2: Assembly of coupler: (a) concept of flat plate and (b) concept of carved plate.

Figure 3(a)). The bottom side is also cut with an angle to the symmetrical axis of the CTPCs. This angle tilts the CTPCs as they sit on the plane, which reduces the entrance angle of transmitted light. The upper side of the CTPCs is carved into a cone shape and extended to form a cylinder. The cylindrical part of the CTPCs could be aligned easily with the fiber optics and spliced to the core of the fiber. A customized adapter with mating sleeve, ferrule, and epoxy is used for connecting CTPC to fiber optics. Using dense array of CTPCs instead of one cone or CPC increases the rim angle whereas it reduces the loss by connecting one fiber to one CTPC instead of connecting bundle of fibers to one cone or CPC. The vertical CTPC, shown in red, is located at the center of the plate. Four CTPCs, shown in yellow, are positioned at the side of plate at the same angle, and four more CTPCs, shown in green, are placed at the same angle at the four corners of the flat plate.

In the second concept, the plate is carved into square surfaces with different angles; therefore, the bottom sides of the CTPCs are perpendicular to the symmetrical axis. This structure is shown in Figure 2(b). As can be seen in Figure 3(b), unlike the first approach, the plate is not flat and the CTPCs stand on the carved surface with no empty space. However, according to derived simulations, both concepts have the same results. The coupler could be manufactured using grinding process and molding. CTPCs may be produced and then assembled on the plate. In this case, proper glue is needed in assembly process. By using the extra numbers of CTPCs, the total surface area of the cores connected to the coupler increases, and thus the coupler could be used for larger primary concentrators. An investigation into the cost effectiveness of such a system is needed in order to optimize the size of the primary concentrator for different applications. In experiment, perfect alignment of the fiber cores will reduce the loss. On the other hand, smoothness of the tip of CTPCs is crucial for reduction of loss. furthermore, the error in angle between CTPCs decrease the efficiency of the coupler. 


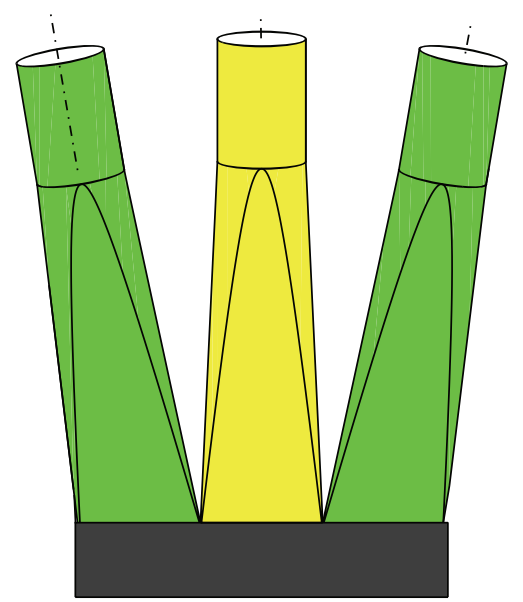

(a)

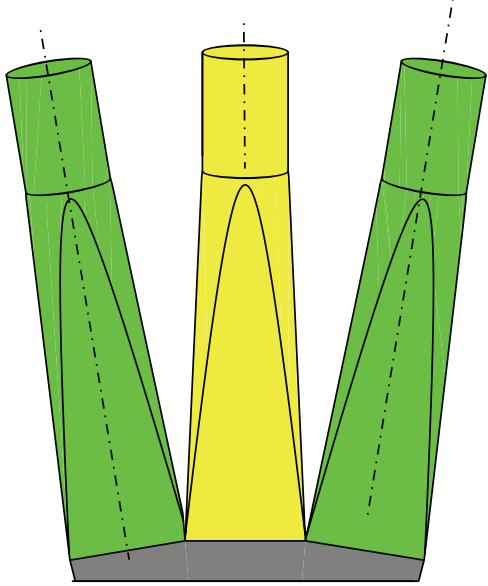

(b)

FIGURE 3: CTPCs cross section: (a) flat design and (b) carved design.

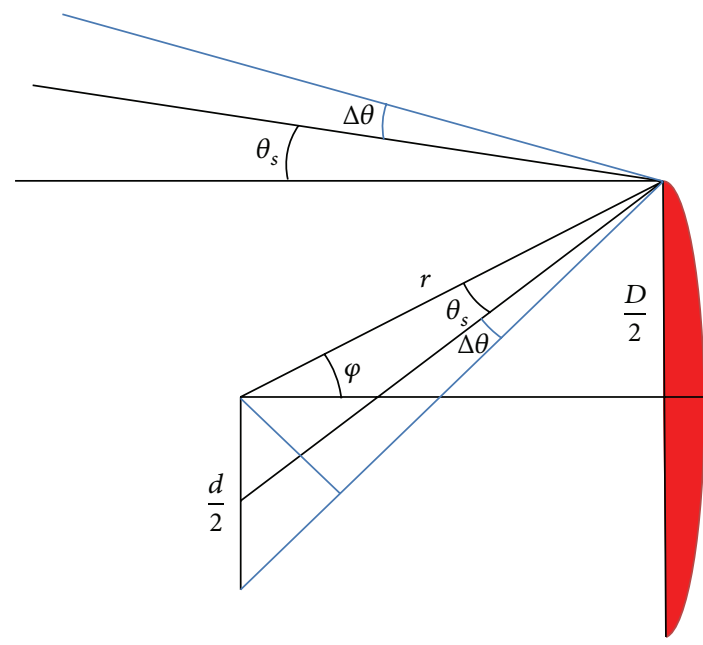

FIGURE 4: Concentrated sun flux by a parabolic dish mirror.

2.1. Plate Design. The inlet light of coupling comes from the primary concentrator. Figure 4 shows the flux of sun that is illuminated on a dish with an aperture of $D$. The rim angle is $\phi$, the sunlight angle is $\theta_{s}$, and the diameter of concentrated light is $d$. An acceptable precision for $\theta_{s}$ is $0.27^{\circ}$, and the occurrence of the probable cumulative errors in the system is given by $\Delta \theta$. The sources of error in the system are an imperfect surface, the structure, movement, alignment, and the sensors of the sun tracker, and so forth. The diameter of concentrated light is given by [26]

$$
d=\frac{D \sin \left(\theta_{s}+\Delta \theta\right)}{\sin \phi \cos \left(\phi+\theta_{s}+\Delta \theta\right)} .
$$

To minimize the diameter of concentrated light, the rim angle is considered to be $45^{\circ}$. Therefore, we can rewrite (1) as

$$
d=\frac{2 D \sin \left(\theta_{s}+\Delta \theta\right)}{\cos \left(\theta_{s}+\Delta \theta\right)-\sin \left(\theta_{s}+\Delta \theta\right)}
$$

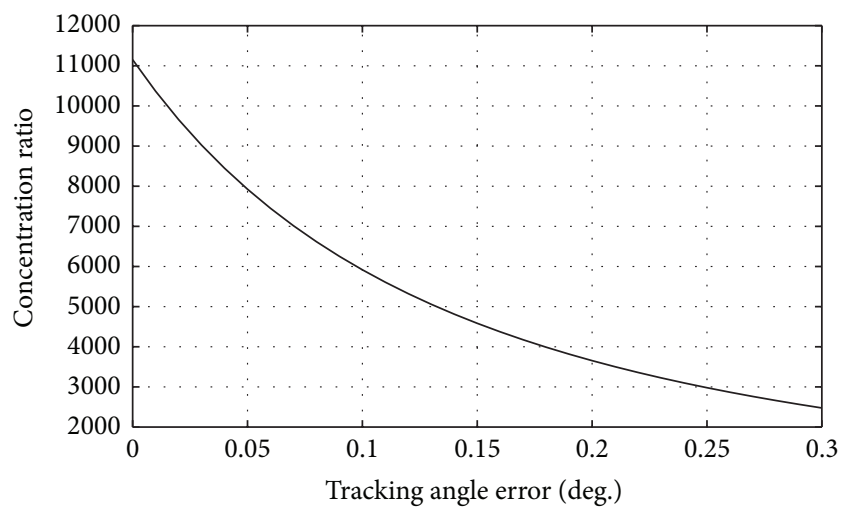

FIGURE 5: Variation of geometrical concentration of a parabolic dish with respect to errors for $45^{\circ}$ rim angle.

Based on the derived diameter and the definition of the geometrical concentration $\left(C=(D / d)^{2}\right)$, we have [27]

$$
C=\frac{1-\sin 2\left(\theta_{s}+\Delta \theta\right)}{4 \sin ^{2}\left(\theta_{s}+\Delta \theta\right)} .
$$

Figure 5 shows a reduction of the geometrical concentration of a parabolic dish as the system error increases from zero to $0.3^{\circ}$. On the other hand, the diameter of the concentrated light increases significantly with increasing errors, which is illustrated in the same figure. Calculation of the concentrated light diameter leads to computation of the dimensions of the plate, with the assumption that the length of the square plate is equal to the focal point diameter.

2.2. CTPC Design. A truncated cone is a simple shape that is widely used as a coupler located between the first concentrator and the fiber optics/photocells. In comparison with compound parabolic concentrators (CPCs), it shows better performance than cones; however, producing CPCs is more difficult and expensive. Williamson [28] illustrated a 


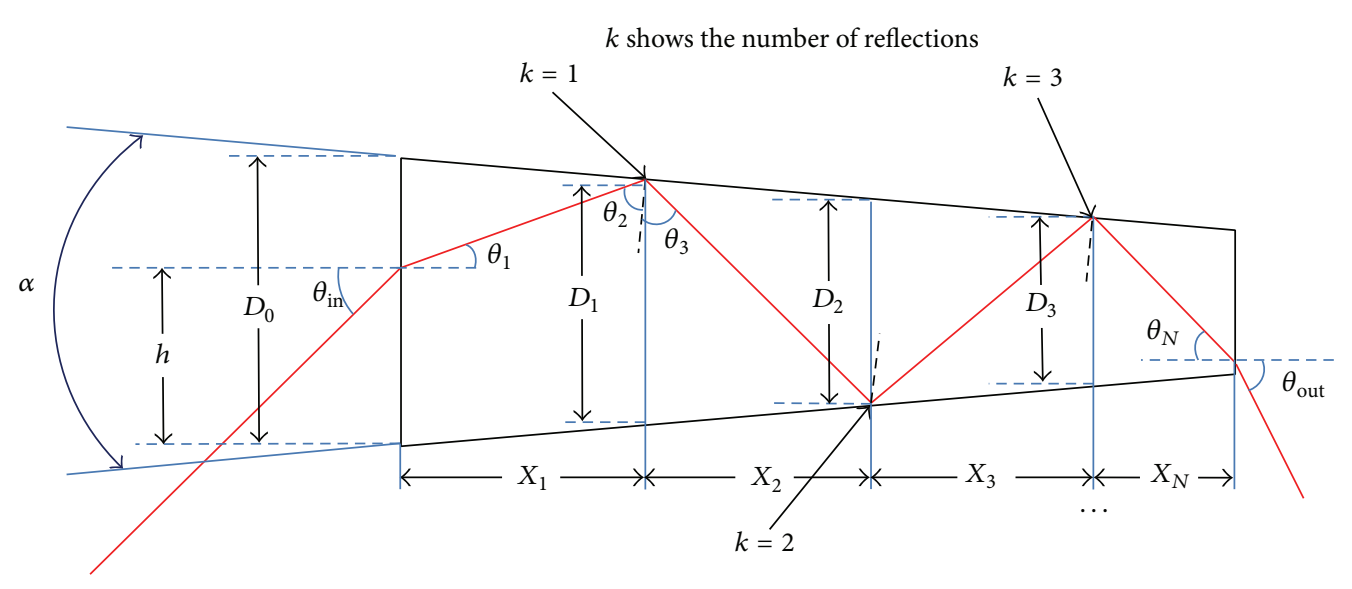

FIGURE 6: CTPC with total internal reflection and the dimensions of reflection points.

geometrical method of ray tracing in a simple cone, and Witte [29] demonstrated that the same procedure is applicable for skew rays. Myer [30] implemented the same method for the concentration of collimated paraxial rays in a cone. Here, we develop a new approach for designing CTPCs for couplers connected to fiber optics. The proposed CTPC design can be divided into two parts. The first part involves the calculation of the maximum amount of total reflection based on the variation of the inlet light and CTPC angles. The second part involves the calculation of CTPC dimensions with respect to the amount of total reflection.

2.2.1. Calculation of CTPC Angle. Figure 6 shows a CTPC and meridian ray with three total internal reflections. Based on Snells law,

$$
n_{1} \sin \theta_{\text {in }}=n_{2} \sin \theta_{1},
$$

where $n_{1}$ is the refraction index of the medium of the inlet ray, $n_{2}$ is the refraction index of the CTPC, $\theta_{\text {in }}$ is the angle of incident light to the forward surface, and $\theta_{1}$ is the refracted angle. To satisfy the total internal reflection condition on the body of CTPC,

$$
\begin{array}{r}
\theta_{2}=\theta_{3} ; \\
\sin \theta_{2}>\frac{n_{1}}{n_{2}},
\end{array}
$$

where the angle of the first incident light to the side wall is $\theta_{2}$ and $\theta_{3}$ is the first total reflection on the side wall of the CTPC. At the first incident light to the side wall of CTPC,

$$
\theta_{2}=\frac{\pi}{2}-\left(\theta_{1}+\frac{\alpha}{2}\right)
$$

where $\alpha$ is the CTPC angle equal to the angle of the pyramid. Substituting (6) into (5) yields

$$
\cos \left(\theta_{1}+\frac{\alpha}{2}\right)>\frac{n_{1}}{n_{2}}
$$

For the number of reflections on the side wall, $k$, with total internal reflections, we have

$$
\theta_{1}<\cos ^{-1}\left(\frac{n_{1}}{n_{2}}\right)-k \frac{\alpha}{2} .
$$

Since an optical fiber with a core and cladding is connected to CTPC, in order to prevent reflection on the end of its surface, we use

$$
\sin \theta_{\text {out }} \leq \sqrt{n_{\text {core }}^{2}-n_{\text {clad }}^{2}}
$$

where $n_{\text {core }}$ and $n_{\text {clad }}$ are the refractive indexes of the core and cladding, respectively. On the other hand, these two fiber parameters can be related to the numerical aperture $(\mathrm{NA}=$ $\left.\sqrt{n_{\text {core }}^{2}-n_{\text {clad }}^{2}}\right)$. The output angle of transmitted light from CTPC is indicated by $\theta_{\text {out }}$. By using the relationship between the internal angles of the designed CTPC in Figure 6, we can obtain

$$
\theta_{N}=\theta_{1}+k \alpha
$$

where $\theta_{N}$ is the incident angle of light to the output surface of CTPC. By applying Snells law for CTPC and optical fiber, we obtain

$$
n_{2} \sin \theta_{N}=n_{\text {core }} \sin \theta_{\text {out }} .
$$

Substituting (11) for (10) and using (9), we find $\theta_{1}$ as

$$
\theta_{1} \leq \sin ^{-1}\left(\frac{n_{\text {core }}}{n_{2}} \sqrt{n_{\text {core }}^{2}-n_{\text {clad }}^{2}}\right)-k \alpha
$$

Figure 7 compares the variations of $\theta_{1}$ in terms of the number of total reflections based on two different relations derived from (8) and (12). The assumed refractive indexes are $n_{1}=1, n_{2}=1.517, n_{\text {core }}=1.459$, and $n_{\text {clad }}=1.378$.

According to Figure 7, there are two different approaches to obtaining the maximum value of $\theta_{1}$ with various angles of CTPC. The upper lines show the mentioned value for the 


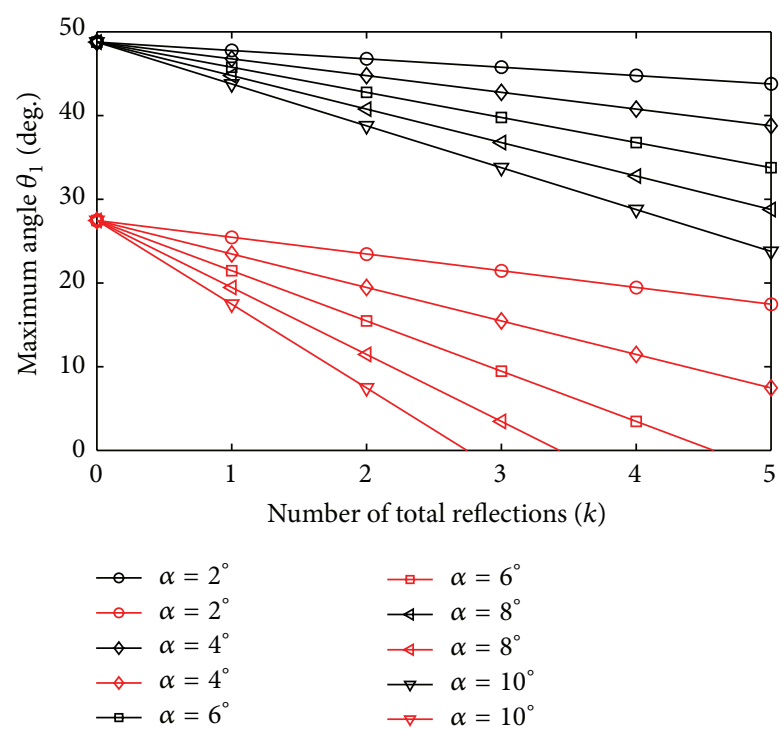

FIGURE 7: Maximum angle of $\theta_{1}$ with respect to the number of total reflections on the body and the end surface (to prevent reflection) for different CTPC angles.

total reflection inside CTPC, whereas the lower lines show the maximum value of $\theta_{1}$ for preventing reflection at the end of CTPC. Connecting the inlet light angle $\theta_{\text {in }}$ to $\theta_{1}$ using (4) and (12) yields

$$
\leq \sin ^{-1}\left[\frac{n_{2} \sin \left(\sin ^{-1}\left(\left(n_{\text {core }} / n_{2}\right) \sqrt{n_{\text {core }}^{2}-n_{\text {clad }}^{2}}\right)-k \alpha\right)}{n_{1}}\right] .
$$

Figure 8 shows the inlet angle versus the maximum number of total reflections on the body of CTPC. For a specific inlet angle $\left(\theta_{\text {in }}\right)$, the number of total reflections $(k)$ decreases with increasing CTPC angle $(\alpha)$.

2.2.2. Calculation of CTPC Dimensions. Figure 9 illustrates a simple general model of CTPC that was indicated in Figure 6. This figure shows the reflected light at two points. The minimum length of CTPC for an optimized number of reflections can be obtained from the summation of distances $\left(X_{k}\right)$ between the points of reflection (i.e., $A$ and $C$ ). The first reflection may occur at any point on the inner surface of CTPC.

To find a relationship between the length of CTPC $(L)$ and the different parameters of the designed CTPC, such as the base lengths for various points of incident light $\left(D_{0}, D_{1}, \ldots, D_{k-1}, D_{k}\right)$ and angle $(\alpha)$, we define $\phi_{k}$ in terms of the refracted angle $\left(\theta_{1}\right)$ and the CTPC angle as

$$
\phi_{k}=\theta_{1}+(k-1) \alpha,
$$

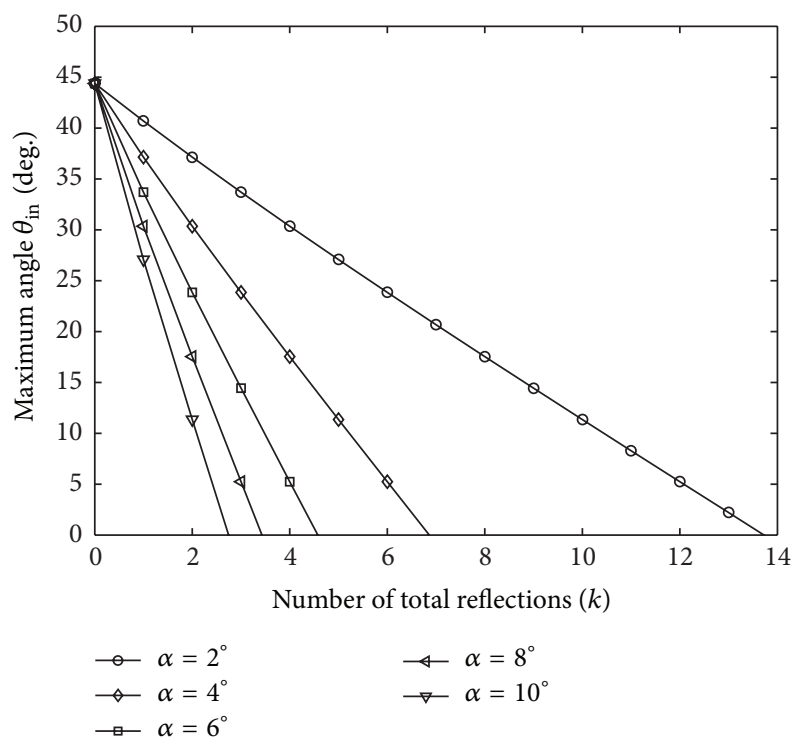

FIGURE 8: Maximum angle of $\theta_{\text {in }}$ with respect to the number of total reflections for different CTPC angles.

where $k$ is the number of reflections and $k \geq 1$. On the other hand, using the tangent formula in the right triangle $A B C$ yields

$$
\tan \phi_{k}=\frac{B C}{A B}=\frac{D_{k-1}-B E}{A B} .
$$

Since the angle $\widehat{B A E}$ is half of the CTPC angle, for the right triangle $A B E$, we have

$$
\tan \frac{\alpha}{2}=\frac{B E}{A B} .
$$

By combining (14) and (15) using (16) and considering $A B=$ $X_{k}$,

$$
\tan \left[\theta_{1}+(k-1) \alpha\right]=\frac{D_{k-1}}{A B}-\tan \frac{\alpha}{2} .
$$

It is clear in Figure 9 for the right triangle $M N P$ that

$$
\tan \theta_{1}=\frac{D_{0}-h-w}{X_{1}}
$$

and that for triangle $P M Q$

$$
\tan \frac{\alpha}{2}=\frac{w}{X_{1}} .
$$

By eliminating $w$ between (18) and (19), we get

$$
X_{1}=\frac{D_{0}-h}{\tan (\alpha / 2)+\tan \theta_{1}} .
$$

In the first reflection (see Figure 6), it is apparent that the first base length $\left(D_{1}\right)$ of CTPC is

$$
D_{1}=D_{0}-2 X_{1} \tan \frac{\alpha}{2} \text {. }
$$




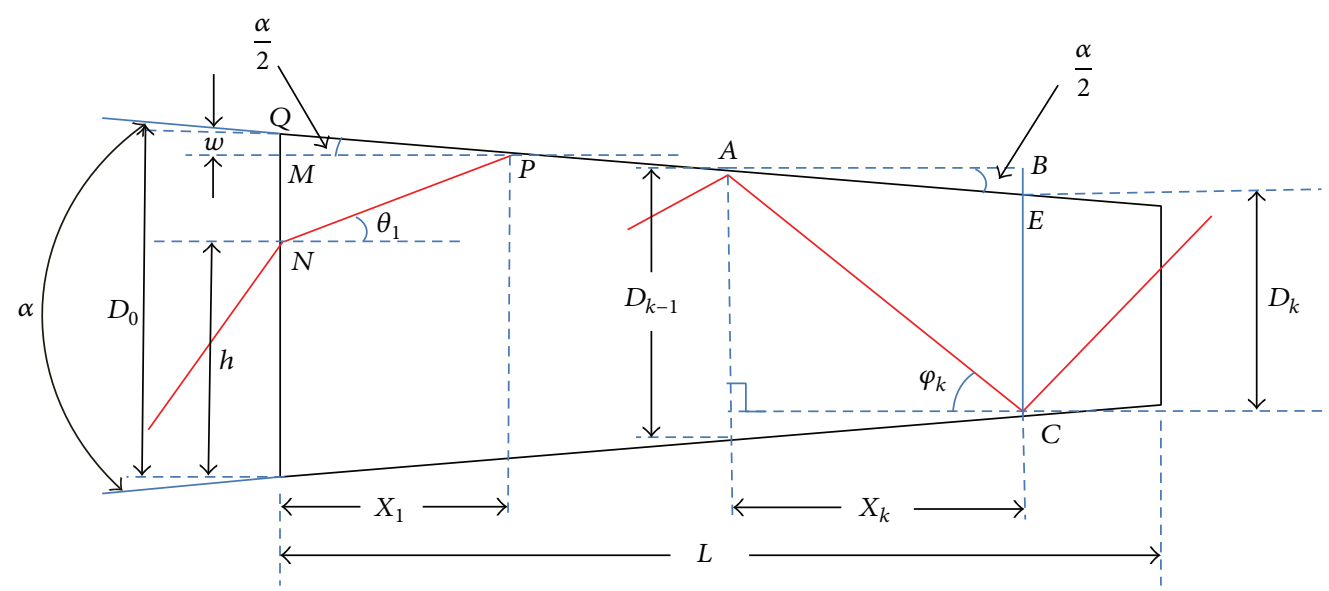

FIGURE 9: Total internal reflection of incident light in a CTPC at $k$ th reflection.

Using a mathematical induction of (20), the distance between reflection points can be derived as follows:

$$
X_{k}=\frac{D_{k-1}}{\tan (\alpha / 2)+\tan \left(\theta_{1}+(k-1) \alpha\right)} ; \quad k \geq 2,
$$

where $D_{k-1}$ can be obtained using the following recurrence relation:

$$
D_{k}=D_{k-1}-2 X_{k} \tan \frac{\alpha}{2} ; \quad k \geq 1
$$

Finally, integrating the above calculated distances allows us to find the length of CTPC as

$$
L \leq \sum_{k=1}^{N} X_{k}
$$

The upper side of CTPC is shaped as a cone (see Figure 3). Therefore, the derived equations are applicable to the cone part as well. The thicker diameter of the cone is the chord of the square base of the pyramid, which reduces the maximum amount of incident light. The maximum concentration of sunlight in the proposed design is limited by the refractive index $\left(n_{\text {core }}\right)$ and sunlight angle $\left(\theta_{s}\right)$ based on the following equation [26]:

$$
C_{\max } \leq \frac{n_{\text {core }}^{2}}{\sin ^{2} \theta_{s}}
$$

Therefore, in designing the coupler, the above limitation of total concentration must be considered.

\section{Coupler Modeling}

A coupler was designed based on the derived equations of Section 2 to design a plate and CTPC. We assumed $900 \mathrm{~W} / \mathrm{m}^{2}$ as the total integrated power intensity of the sun for an AM 1.5 D reference spectrum (ASTMG 173) [31]. The calculated angle between CTPCs was $9.5^{\circ}$. The geometrical concentration of CTPC was 1.97 and the input power of

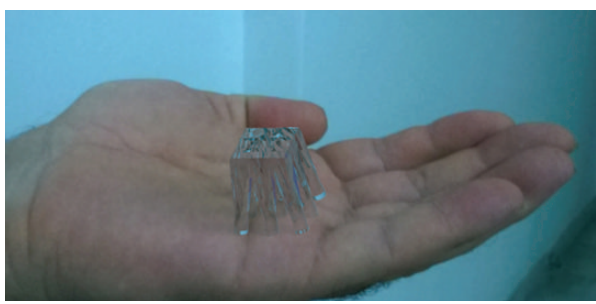

FIGURE 10: CAD prototype of coupler.

the coupler was assumed to be $300 \mathrm{~W}$ for a dish with a $650 \mathrm{~mm}$ aperture diameter. Using CAD modeling software, a 3D model was created to be used in further studies of the experimental model and the fabricating process. Figure 10 shows a CAD prototype of the proposed coupler.

The 3D model was developed using a nonsequential model for ray tracing in Zemax software. The rim angle of the dish was considered to be $45^{\circ}$, which concentrated the light directly onto the coupler. Hence, the multipoint sources of light were assumed to propagate the light with five wavelengths from 400 to $800 \mathrm{~nm}$ in our simulation. The angle of propagation was $45^{\circ}$, and a detector was located behind the point source, whereas each CTPC had a smallerdiameter detector. The coupler was placed a few millimeters from the focal point, and therefore the rays were divergent. In the simulation, $B K 7$ was considered as a material for the coupler. The wavelengths in the simulation were 400, 550, and $700 \mathrm{~nm}$. Figure 11 shows a layout of the coupler with a ray tracing format in shaded and $3 \mathrm{D}$ models. If the diameter of concentrated light and the occurrence of the probable cumulative errors in the system are to be determined, the diameter of the dish could be calculated using (2). As an instance, the aperture of the dish for a coupler with $26.01 \mathrm{~mm}^{2}$ entrance surface and nine fiber optics with $1 \mathrm{~mm}$ diameter of core and total error of $0.1^{\circ}$ would be $392 \mathrm{~mm}$. On a clear day, 900 watts of solar energy per square meter is expected of absorption area. A dish with $1160 \mathrm{~mm}$ aperture is needed for a coupler including 25 CTPCs. Considering the efficiency of the entire system $30 \%$ so 280 watts of electrical output 


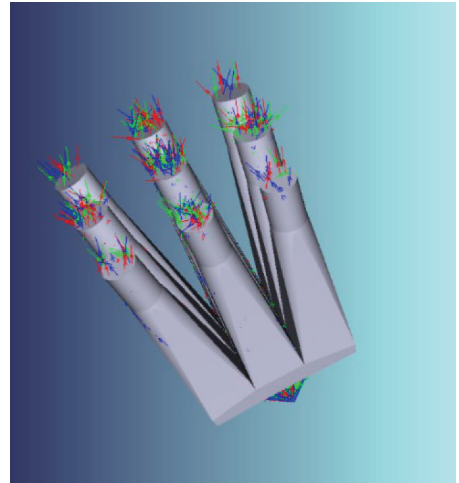

(a)

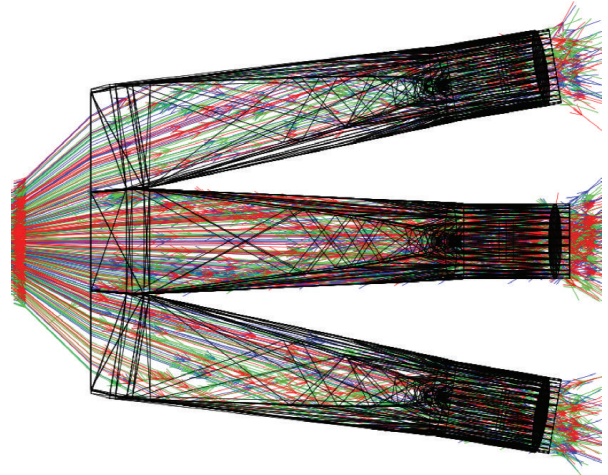

(b)

FIGURE 11: Layout of coupler for ray tracing: (a) shaded model and (b) 3D layout.

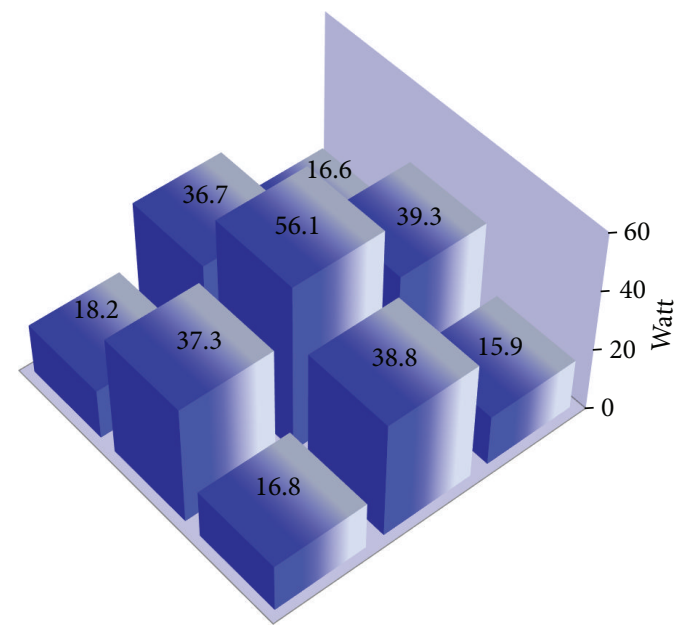

FIGURE 12: Distribution of power amplitudes (in watts) transmitted by CTPCs for a $300 \mathrm{~W}$ input.

would be available from one dish. Take into account 1$3 \mathrm{kw}$ for a typical house, which would require 4-12 dishes. Depending on the site, the array of dishes can be mounted on the rooftop or on the ground near the building. Totally, 100-300 fiber optics with one millimeter diameter of core is required, respectively, for $1-3 \mathrm{kw}$ output, bearing in mind that the length of fibers used for generating electricity is less than the fibers used for daylighting. The cost of fiber optics is dominated by the production process, and only (around 10\%) small fraction accounts for material. If we assume that the production cost is the same as common mass-produced single mode silica fiber with outer diameter of $0.125 \mathrm{~mm}(\$ 0.1 / \mathrm{m})$ and allocates the cost of the fused silica $(\$ 250 / \mathrm{kg})$, the cost would be $(\$ 0.53 / \mathrm{m})$ for fiber optics with $1 \mathrm{~mm}$ diameter of core. So it is reasonable to assume that if massive demand arises, then the cost of fiber optics for $1-3 \mathrm{kw}$ will be \$590-\$1760 for the couplers with 25 CTPCs.

\section{Simulation Results and Discussion}

The efficiency of the designed coupler under noncollimate irradiation, the angle of inlet light into the fiber optics, and the propagated light intensity via the components were investigated by ray tracing. This efficiency was calculated by dividing the total occupied powers by CTPCs to the light source power through the following equation:

$$
\text { eff }=\frac{\sum_{i=1}^{n} P_{i}^{T}}{P_{\text {in }}},
$$

where $P^{T}$ is the power transmitted by each fiber optic, $P_{\text {in }}$ is the total inlet power of the coupler, and $n$ is the number of CTPCs. Figure 12 shows the power transmitted by each CTPC while $300 \mathrm{~W}$ power was applied by multipoint sources. Although there was significant variation between the power transmitted by CTPCs on the corners, sides, and center, the amounts of power transmitted by the CTPCs that were positioned on the sides or corners were close to each other.

The efficiency of the designed coupler was $92 \%$ in the simulation. Fresnel reflection on the surfaces of the optical components caused a loss of power. In this coupler, Fresnel reflection only occurred on the flat plate surface. Incoherent irradiance of the transmitted light and the distribution of radiant intensity by CTPCs are shown in Figure 13 for $10 \mathrm{~W}$ 

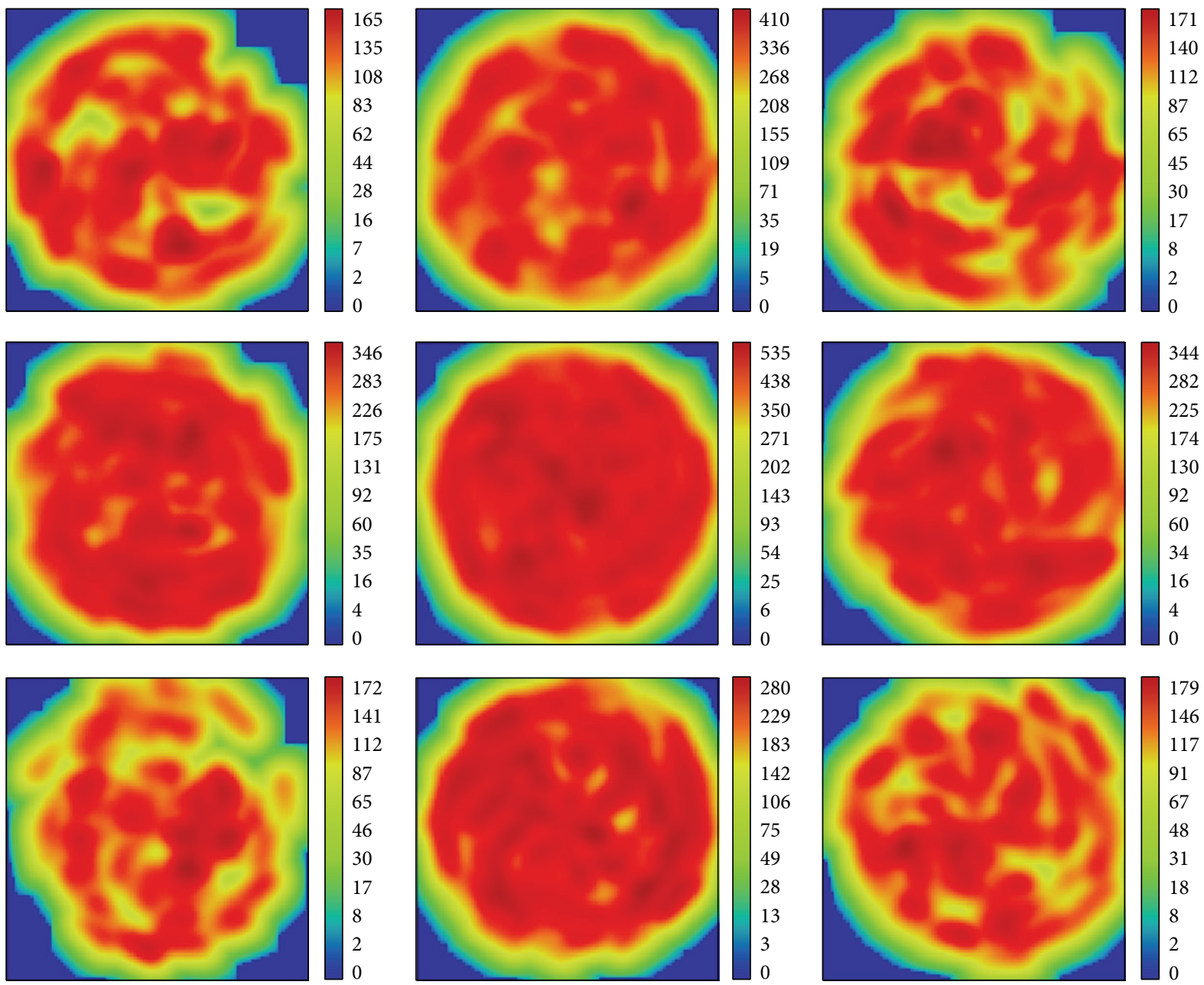

FIGURE 13: Distribution of radiant intensity for CTPCs according to their position.

source power. The maximum intensity of light was exhibited by the centrally placed CTPC and the least intensity of transmitted light was exhibited by CTPCs positioned on the corners of the plate. The loss due to Fresnel reflection on the tip of fiber optics and the loss of connector were not taken into account in calculating the efficiency of coupler. Considering $4 \%$ loss as normal reflection on fiber tip and $11 \%$ $(0.5 \mathrm{~dB})$ in connecting multimode fiber to the coupler, the efficiency reduces to $77 \%$. Refractive index of BK7 reduces from 1.5308 at $400 \mathrm{~nm}$ to 1.5108 at $800 \mathrm{~nm}$ [32]. The efficiency of the coupler reduced while the wavelength increased. The reduction of efficiency was less than $1.5 \%$.

The angle of inlet light into the fiber optics plays a key role in reducing the light leakage of fiber optics in the transmission of sunlight [33]. Figure 14 compares the angular range and the amplitude of light intensity for CPTCs. The coupler was designed for a fiber with a numerical aperture (NA) of 0.48 , which means that the maximum acceptable cone angle of the fiber should be $56^{\circ}$. The simulated results for angular range demonstrate the validity of the calculations for the designed coupler. As shown in Figure 13, all CTPCs around center one have less uniform distribution of intensity. Hence, the center CTPC could be used for electricity generation via multijunction solar cells due to its uniform intensity and small angular range of radiation, that is, less than $30^{\circ}$ (See Figure 14). The uniformity for other CTPCs that are used for lighting could be increased by grouping the fiber optics according to their intensity and by combining the sunlight and the LED light with a hybrid luminaire.

\section{Conclusion}

In this study, a new design of coupler for multifunctional solar applications was presented. This design includes a plate (flat or carved) and nine CTPCs. The shape of CTPCs provided an easy connection between the fiber and the coupler, such that there was no space in the assembly of CTPCs on the plate surface. Also, the coupler could be connected to a highconcentration parabolic dish and fiber optics while being used as a secondary concentrator. The theory for designing and modeling CTPCs, as well as the related mathematical equations for the coupler structure, has been developed. Simulation of the proposed coupler showed 92\% efficiency, and the rim angle of the parabolic dish was maintained at $45^{\circ}$. The obtained results indicate that the peak of radiant intensity and the total power of the corner CTPCs noticeably decrease the 

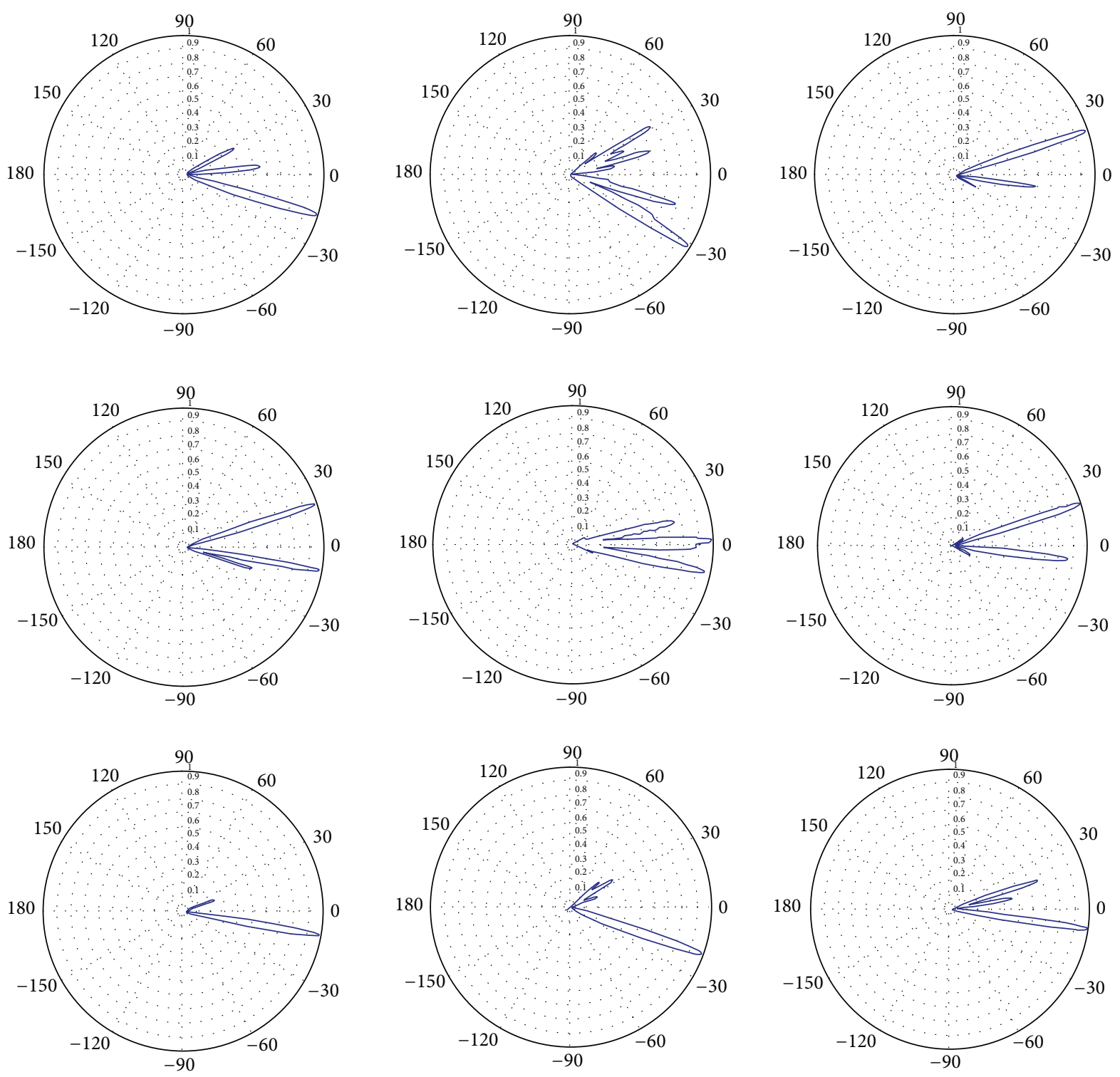

FIGURE 14: Distribution of angular range and amplitude for CTPCs according to their position.

amplitude of power intensity. The simulated results confirm that the central CTPC is appropriate for generating electric power using multijunction solar cells; the CTPCs located at the side of the plate are suitable for producing heat, whereas those at the corners can be used to provide daylighting.

\section{Competing Interests}

The authors declare that they have no competing interests.

\section{Acknowledgments}

This work has been supported by the University of Malaya under the High Impact Research (Grant no. UM.0000005/ HIR.C1), the UMRG (Grant no. RP021A-13AET), and the PPP (Grant no. PG218-2015A). The authors would like to thank Dr.
Mohd Haniff Ibrahim from the photonics lab and Universiti Teknologi Malaysia for providing access to Zemax software.

\section{References}

[1] S. Pless and P. Torcellini, Net-Zero Energy Buildings: A Classification System Based on Renewable Energy Supply Options, 2010.

[2] G. Mittelman, A. Kribus, and A. Dayan, "Solar cooling with concentrating photovoltaic/thermal (CPVT) systems," Energy Conversion and Management, vol. 48, no. 9, pp. 2481-2490, 2007.

[3] J. M. Cariou, J. Dugas, and L. Martin, “Transport of solar energy with optical fibres," Solar Energy, vol. 29, no. 5, pp. 397-406, 1982.

[4] W. Grisé and C. Patrick, "Passive solar lighting using fiber optics," Journal of Industrial Technology, vol. 19, no. 1, pp. 1-7, 2002. 
[5] D. Feuermann and J. M. Gordon, "Solar surgery: remote fiber optic irradiation with highly concentrated sunlight in lieu of lasers," Optical Engineering, vol. 37, no. 10, pp. 2760-2767, 1998.

[6] S. Amara, Novel and ancient technologies for heating and cooling buildings [Ph.D. thesis], 2011.

[7] C. Zidani, B. Benyoucef, and N. Madini, "Optimization of photothermal system based on the idea of transmission solar energy via optical fibres," Physics Procedia, vol. 55, pp. 493502, 2014, Proceedings of the 8th International Conference on Material Sciences, CSM8-ISM5.

[8] A. Kribus, O. Zik, and J. Karni, "Optical fibers and solar power generation," Solar Energy, vol. 68, no. 5, pp. 405-416, 2000.

[9] F. C. Christo, "Numerical modelling of wind and dust patterns around a full-scale paraboloidal solar dish," Renewable Energy, vol. 39, no. 1, pp. 356-366, 2012.

[10] C. Kandilli, K. Ulgen, and A. Hepbasli, "Exergetic assessment of transmission concentrated solar energy systems via optical fibres for building applications," Energy and Buildings, vol. 40, no. 8, pp. 1505-1512, 2008.

[11] T. Nakamura, B. Comaskey, and M. Bell, "Development of optical components for space-based solar power system for ISRU and regnerative life support," in Proceedings of the 40th AIAA Aerospace Sciences Meeting and Exhibit, January 2002.

[12] D. Feuermann and J. M. Gordon, "Solar fiber-optic mini-dishes: a new approach to the efficient collection of sunlight," Solar Energy, vol. 65, no. 3, pp. 159-170, 1999.

[13] A. L. Luque and A. Viacheslav, Concentrator Photovoltaics, vol. 130 of Springer Series in Optical Sciences, Springer, Berlin, Heidelberg, 2007.

[14] K. Lovegrove, G. Burgess, and J. Pye, "A new $500 \mathrm{~m}^{2}$ paraboloidal dish solar concentrator," Solar Energy, vol. 85, no. 4, pp. 620-626, 2011.

[15] L. Li and S. Dubowsky, "A new design approach for solar concentrating parabolic dish based on optimized flexible petals," Mechanism and Machine Theory, vol. 46, no. 10, pp. 1536-1548, 2011.

[16] X.-L. Xia, G.-L. Dai, and Y. Shuai, "Experimental and numerical investigation on solar concentrating characteristics of a sixteendish concentrator," International Journal of Hydrogen Energy, vol. 37, no. 24, pp. 18694-18703, 2012.

[17] C. Ciamberlini, F. Francini, G. Longobardi, M. Piattelli, and P. Sansoni, "Solar system for exploitation of the whole collected energy," Optics and Lasers in Engineering, vol. 39, no. 2, pp. 233246, 2003.

[18] M. A. Soderstrand, S. B. Lee, and P. Chung, "Mini-dish based hybrid Concentrated Solar Power (CSP) system for home use," in Proceedings of the IEEE 56th International Midwest Symposium on Circuits and Systems (MWSCAS '13), pp. 689692, Columbus, Ohio, USA, August 2013.

[19] D. Liang, L. Fraser Monteiro, M. Ribau Teixeira, M. L. Fraser Monteiro, and M. Collares-Pereira, "Fiber-optic solar energy transmission and concentration," Solar Energy Materials and Solar Cells, vol. 54, no. 1-4, pp. 323-331, 1998.

[20] R. Winston and E. Ning, Nonimaging Radiant Energy Device, 1996.

[21] D. Feuermann, J. M. Gordon, and M. Huleihil, "Solar fiberoptic mini-dish concentrators: first experimental results and field experience," Solar Energy, vol. 72, no. 6, pp. 459-472, 2002.

[22] K. He, H. Zheng, Z. Li, Taotao, and J. Dai, "Design and investigation of a novel concentrator used in solar fiber lamp," Solar Energy, vol. 83, no. 11, pp. 2086-2091, 2009.
[23] I. Ullah and S. Shin, "Highly concentrated optical fiber-based daylighting systems for multi-floor office buildings," Energy and Buildings, vol. 72, pp. 246-261, 2014.

[24] X. Yu, Y. Su, H. Zheng, and S. Riffat, "A study on use of miniature dielectric compound parabolic concentrator (dCPC) for daylighting control application," Building and Environment, vol. 74, pp. 75-85, 2014.

[25] G. E. Arnaoutakis, J. Marques-Hueso, T. K. Mallick, and B. S. Richards, "Coupling of sunlight into optical fibres and spectral dependence for solar energy applications," Solar Energy, vol. 93, pp. 235-243, 2013.

[26] A. Rabl, "Comparison of solar concentrators," Solar Energy, vol. 18, no. 2, pp. 93-111, 1976

[27] A. Rabl, Active Solar Collectors and Their Applications, Oxford University Press, 1985.

[28] D. E. Williamson, "Cone channel condenser optics," Journal of the Optical Society of America, vol. 42, no. 10, pp. 712-714, 1952.

[29] W. Witte, “Cone channel optics," Infrared Physics, vol. 5, no. 4, pp. 179-185, 1965.

[30] J. H. Myer, "Collimated radiation in conical light guides," Applied Optics, vol. 19, no. 18, pp. 3121-3123, 1980.

[31] American Society for Testing and Materials (ASTM), "Reference solar spectral irradiance," ASTM G 173-03, NREL, 2012.

[32] M. Polyanskiy, "Refractive index database," http://refractiveindex.info.

[33] D. Feuermann, J. M. Gordon, and M. Huleihil, "Light leakage in optical fibers: experimental results, modeling and the consequences for solar concentrators," Solar Energy, vol. 72, no. 3, pp. 195-204, 2002. 

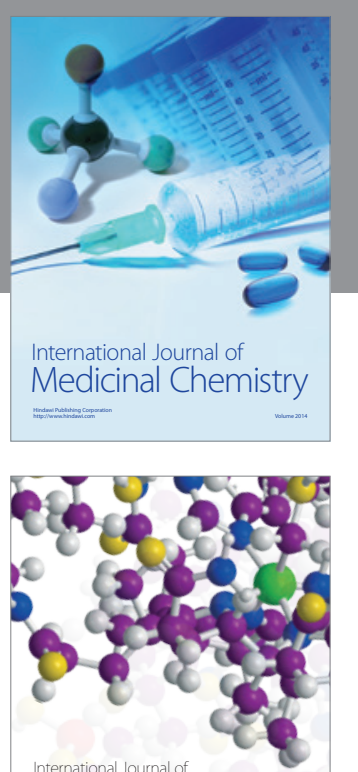

Carbohydrate Chemistry

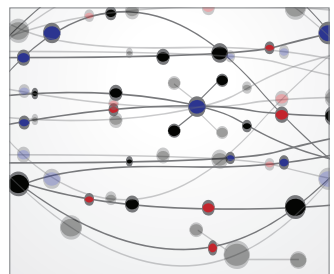

The Scientific World Journal
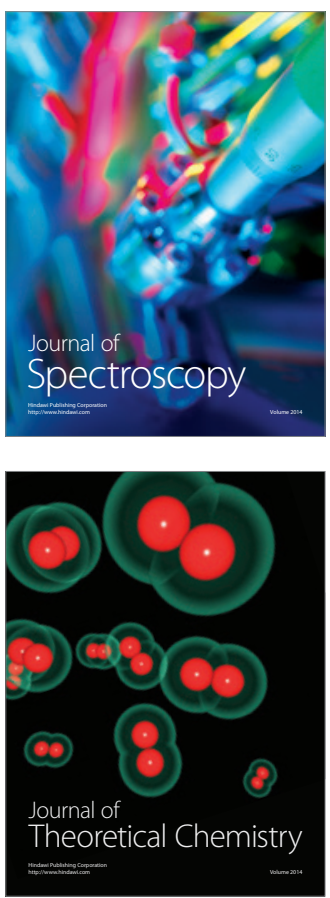
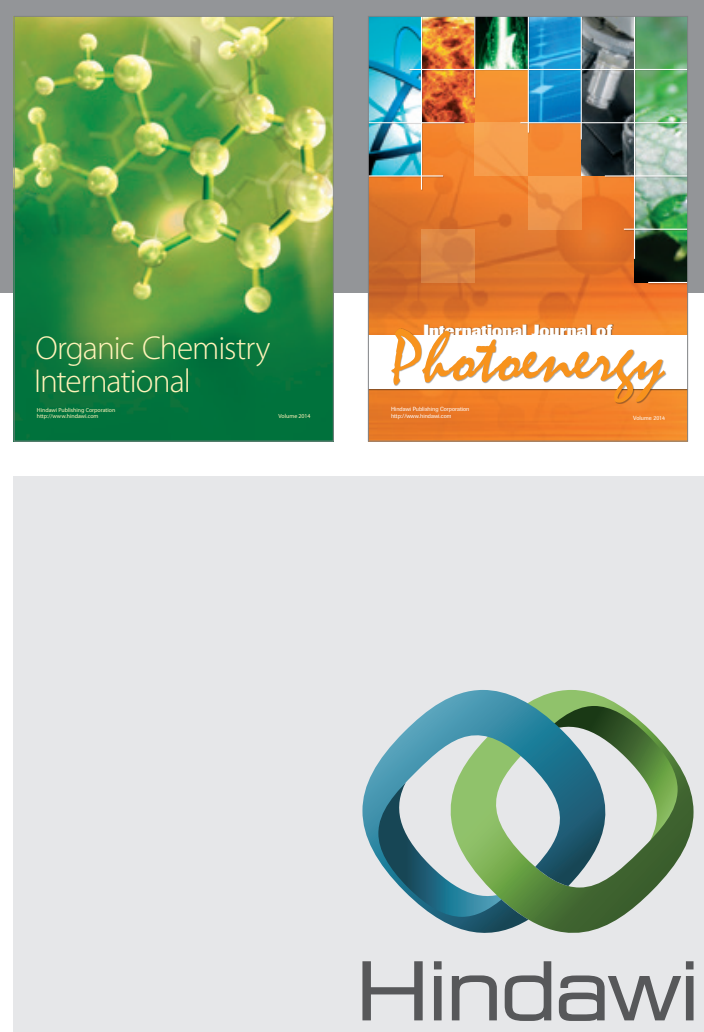

Submit your manuscripts at

http://www.hindawi.com

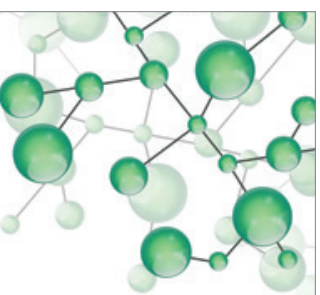

International Journal of

Inorganic Chemistry

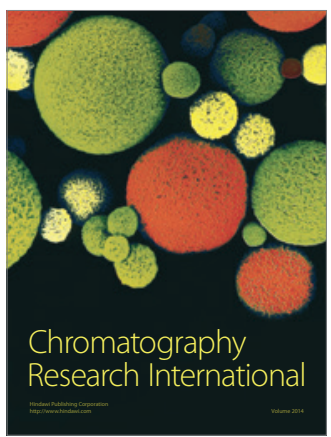

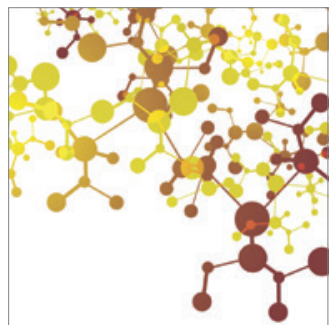

Applied Chemistry
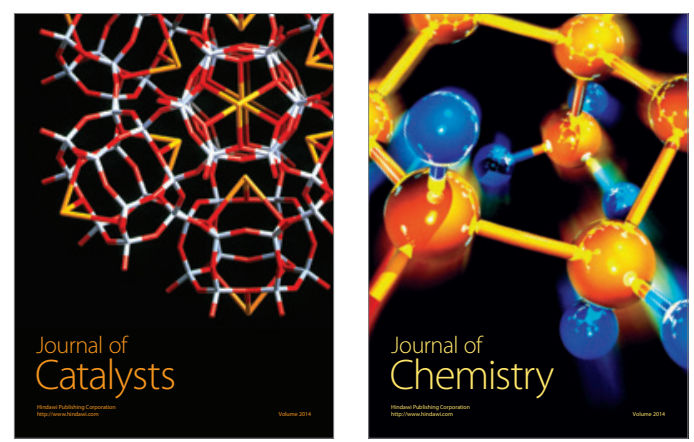
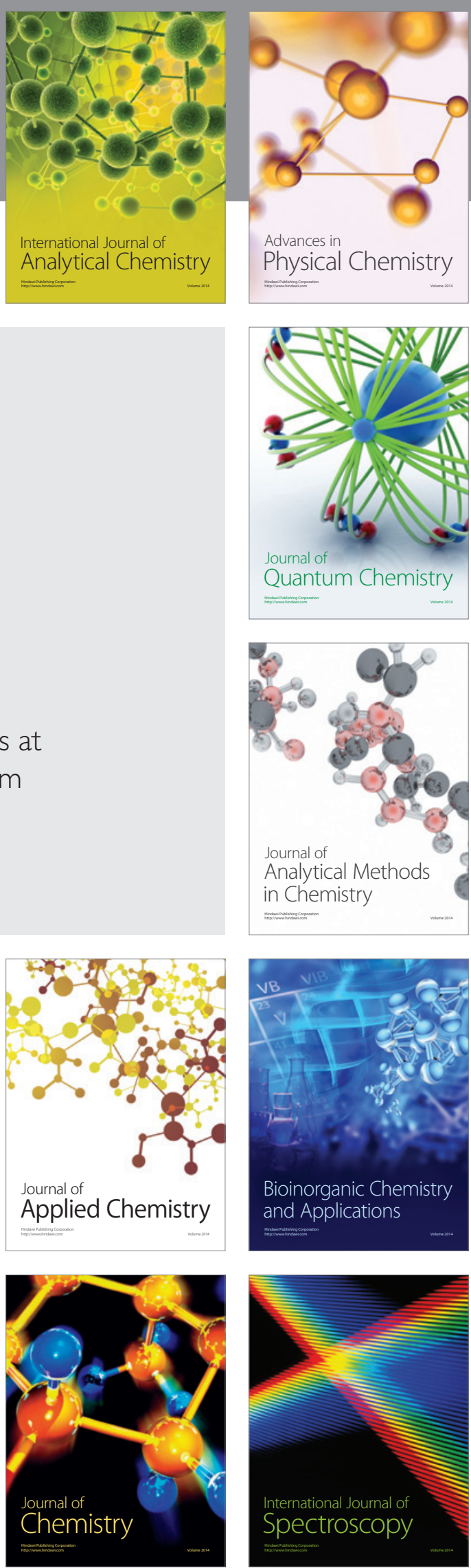